\title{
Active acoustic cloaking in a convected flow field
}

Daniel Eggler, Mahmoud Karimi, and Nicole Kessissoglou

Citation: The Journal of the Acoustical Society of America 146, 586 (2019); doi: 10.1121/1.5119225

View online: https://doi.org/10.1121/1.5119225

View Table of Contents: https://asa.scitation.org/toc/jas/146/1

Published by the Acoustical Society of America

\section{ARTICLES YOU MAY BE INTERESTED IN}

Extensions to the acoustic scattering analysis for cloaks in non-uniform mean flows

The Journal of the Acoustical Society of America 146, 41 (2019); https://doi.org/10.1121/1.5115046

Design of an acoustic illusion device based on a shifting medium and multi-folded transformation

The Journal of the Acoustical Society of America 146, 505 (2019); https://doi.org/10.1121/1.5118244

Introduction to the special issue on non-reciprocal and topological wave phenomena in acoustics

The Journal of the Acoustical Society of America 146, 719 (2019); https://doi.org/10.1121/1.5119133

Observations of phase and intensity fluctuations for low-frequency, long-range transmissions in the Philippine Sea and comparisons to path-integral theory

The Journal of the Acoustical Society of America 146, 567 (2019); https://doi.org/10.1121/1.5118252

Effect of a serrated trailing edge on sound radiation from nearby quadrupoles

The Journal of the Acoustical Society of America 141, 2997 (2017); https://doi.org/10.1121/1.4982201

Acoustic scattering for 3D multi-directional periodic structures using the boundary element method The Journal of the Acoustical Society of America 141, 313 (2017); https://doi.org/10.1121/1.4973908

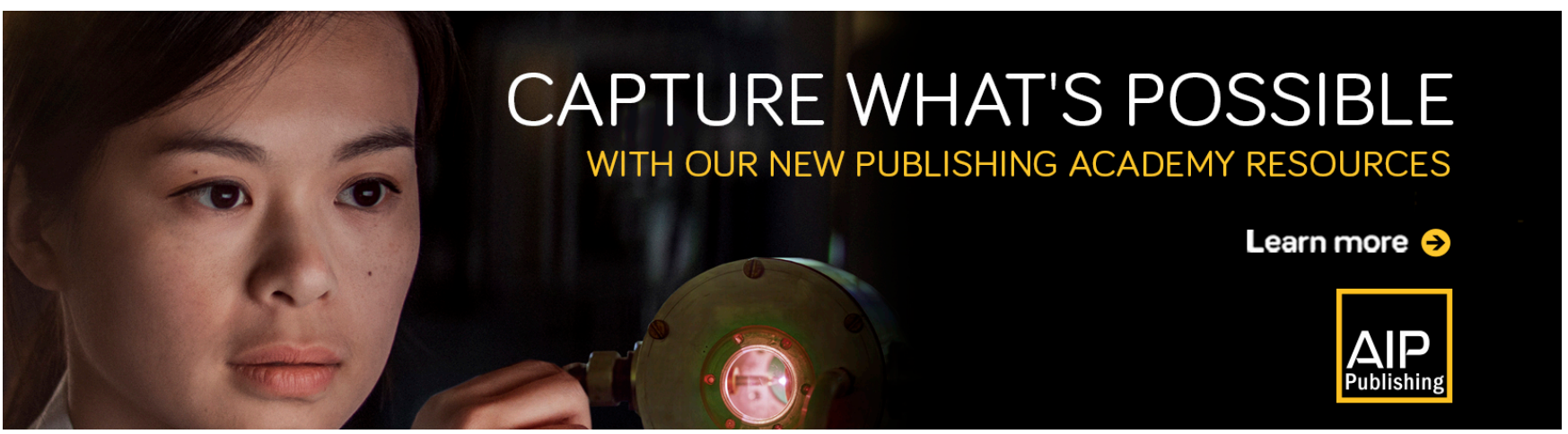




\title{
Active acoustic cloaking in a convected flow field
}

\author{
Daniel Eggler, ${ }^{1, a)}$ Mahmoud Karimi, ${ }^{2, b)}$ and Nicole Kessissoglou ${ }^{1}$ \\ ${ }^{1}$ School of Mechanical and Manufacturing Engineering, The University of New South Wales, Sydney, \\ New South Wales, Australia \\ ${ }^{2}$ Centre for Audio, Acoustics and Vibration, University of Technology Sydney, New South Wales, Australia
}

(Received 3 April 2019; revised 13 June 2019; accepted 9 July 2019; published online 29 July 2019)

Acoustic cloaking has mostly been considered within a stationary fluid. The authors herein show that accounting for the effects of convection in the presence of fluid flow is critical for cloaking in the acoustic domain. This work presents active acoustic cloaking in a convected flow field for two different incident fields, corresponding to a plane wave and a single monopole source, impinging on a rigid body. Monopole control sources circumferentially arranged around the rigid body are used to generate a secondary acoustic field to destructively interfere with the primary scattered field arising from the incident excitation cases. The authors show that for sound waves in a moving fluid, active cloaking can only be achieved using a convected cloak, which is dependent on Mach number. (C) 2019 Acoustical Society of America. https://doi.org/10.1121/1.5119225

Pages: 586-594

\section{INTRODUCTION}

The last decade has seen a surge of interest in the field of cloaking, commencing when Pendry et al. ${ }^{1}$ detailed a method using transformation optics to guide electromagnetic waves around an object to render it invisible, thus signalling the beginning of passive cloaking. Cloaking in electromagnetics, ${ }^{2-6}$ acoustics, ${ }^{7-35}$ and elastodynamics ${ }^{36-40}$ was soon realized. Cloaking methods in acoustics can be broadly categorized into two types: passive and active. Passive acoustic cloaks are designed for either a predetermined frequency ${ }^{7-14}$ or a broad frequency range ${ }^{15-23}$ using metamaterials, comprising structures containing materials with anisotropic properties that guide an incident field around an object. Torrent and Sanchez-Dehesa ${ }^{10}$ proposed a circular multilayered composite that utilized two types of isotropic metamaterials to acoustically cloak a two-dimensional (2D) domain. Popa and Cummer ${ }^{14}$ proposed an alternative acoustic cloaking design that comprised homogeneous anisotropic layers. A broadband acoustic ground cloak constructed using homogeneous perforated plastic plates was experimentally demonstrated for two- and three-dimensions. ${ }^{18,19}$ Farhat et al. ${ }^{21}$ investigated broadband acoustic cloaking of linear surface liquid waves. They experimentally presented a structured metamaterial cloak comprising curved metallic sectors that concealed a rigid object from incident surface waves due to an acoustic source. Chen et al. ${ }^{22,23}$ presented a novel design of pentamode materials submerged in water to generate a $2 \mathrm{D}$ solid cloak capable of broadband frequency performance. Unlike the aforementioned passive cloaks, the surrounding fluid medium did not pass through the cloak structure. By taking advantage of solid pentamode materials to mimic metafluids with highly anistropic moduli, they were able to experimentally and numerically demonstrate cloaking of an object enclosed by the solid cloak.

\footnotetext{
${ }^{a}$ Electronic mail: d.eggler@unsw.edu.au

b) Also at: School of Mechanical and Manufacturing Engineering, The University of New South Wales, Australia.
}

Active acoustic cloaks may be exterior or interior, using control sources respectively located external to or within the cloaked region. Active exterior cloaks generate a cloaked region enclosing the scatterer, which is independent of the scatterer topology. Exterior cloaking has been demonstrated for acoustic cloaking of a body within an infinite fluid, ${ }^{24-26}$ as well as elastodynamic cloaking of inclusions from a flexural wave field in an infinite plate. ${ }^{36,37}$ Guevara Vasquez et $a l^{24,25}$ analytically proposed $2 \mathrm{D}$ and three-dimensional exterior acoustic cloaks using multipole sources to generate the cloaked region. Norris et al. ${ }^{36}$ generalized a 2D elastodynamic cloak while Futhazar et al. ${ }^{37}$ described how to efficiently obtain control source coefficients to effectively cloak a region within a Kirchhoff plate.

Active interior cloaks do not possess such scatterer independency as the cloaking process is implemented after the incident field has interacted with the scatterer. The acoustic field before implementation of cloaking is commonly termed the primary field. Control sources are used to generate a secondary acoustic field. The residual field, resulting from destructive interference between the primary and secondary fields, resembles the incident field. Interior cloaking has been applied to both acoustic ${ }^{27-35}$ and elastic ${ }^{38-40}$ domains. Bobrovnitskii ${ }^{27,28}$ proposed a novel method where cloaking was achieved by covering the body with a smart skin containing structural actuators, structural sensors, and pressure sensors capable of inducing surface impedances such that the body becomes non-scattering. Rajabi and Mojahed ${ }^{29}$ analytically demonstrated acoustic cloaking in the backscatter region with a shell that was stimulated by a radially polarized piezoelectric bonded layer. Conventional active noise control (ANC) techniques as pioneered by Nelson and Elliot $^{41}$ that require a priori knowledge of the incident field have also been implemented for acoustic cloaking. Cheer ${ }^{30}$ applied conventional ANC techniques to non-trivial shapes, where he demonstrated acoustic cloaking of a rectangular cuboid by employing control sources (loudspeakers) and error sensors (microphones). Similarly, conventional ANC 
techniques have also been implemented to acoustically cloak rigid and elastic cylindrical shells. ${ }^{31}$ Active structural acoustic cloaking was introduced, whereby control forces directly applied to the shell were driven to modify the structural responses such that the resultant structure-borne sound field generated the cloaked field. Friot $e t a l{ }^{32,33}$ devised a method using two layers of error sensors to mitigate the requirement of a priori knowledge in order to experimentally demonstrate cloaking of one-dimensional ${ }^{32}$ and three-dimensional ${ }^{33}$ objects with conventional ANC techniques. O'Neill et al. ${ }^{38}$ analytically investigated cloaking of an inclusion within an infinite plate from an incident flexural wavefield. This work was extended to further consider resonant coated inclusions ${ }^{39}$ as well as a finite cluster of pins. ${ }^{40}$ An advantage of active cloaking applicable to both exterior and interior approaches is that controller parameters can be updated in real time to adjust for variation in frequency and incident field behaviour.

The aforementioned cloaking studies were conducted in a stationary fluid medium. However, when the fluid velocity is non-zero, deterioration of the cloaking performance of both passive and active cloaks is observed. A passive cloak for sound waves in a moving fluid that accounted for potential flow effects was recently demonstrated by Huang et al. ${ }^{42}$ It was shown that when fluid flow was not taken into account, the performance of an acoustic cloak decreased with increasing Mach number and increasing frequency. It was also found that a distinct shadow zone remained downstream of the scatterer despite a convected formulation. Huang et $\mathrm{ll} .{ }^{43}$ further examined the performance of a passive acoustic cloak in the presence of a turbulent flow field. Ryoo and Jeon ${ }^{44}$ successfully designed and simulated a passive acoustic cloak that considered the additional effects of fluid compressibility and non-uniformity in the convected flow field. While the shadow zone was minimized with this new formulation, it was not completely eliminated. Iemma and Palma ${ }^{45}$ recently proposed a method for convective correction of a metamaterial cloak in flow initially designed for a stationary fluid. By investigating the location of a monopole source relative to the convected acoustic cloak, they analytically and numerically determined that the shadow zone could be significantly minimised for a monopole located orthogonal to both the direction of flow and the metamaterial cloak. The convected metamaterial cloak exhibited the strongest shadow zone when the monopole and metamaterial cloak were aligned with the direction of flow.

We herein present an active acoustic cloak that accounts for the effects of a convected flow field. Our active control strategy is based on discrete frequency control and requires a priori knowledge of the incident excitation. Minimisation of a cost function that optimizes the control source amplitudes is implemented to produce the desired acoustic field at the error sensors. The cost function is a quadratic function in terms of the transfer functions for the primary and secondary acoustic fields, respectively, arising from scattering by the rigid body due to the incident field (plane wave or single monopole source) and each of the monopole control sources. Results comparing the performance of the active acoustic cloak when convected flow is included or neglected in the formulation are presented. The layout of this paper is as follows. Section II presents the mathematical framework for the acoustic transfer functions due to scattering by a rigid cylinder under incident plane wave and monopole source excitation, as well as the coordinate transformation method that introduces the effects of potential flow to the acoustic domain. Section III describes the active control configuration and method. Section IV presents the results and discussion and Sec. V summarises the main findings.

\section{FORMULATION OF THE PHYSICAL SYSTEM}

\section{A. Acoustic model}

A brief overview of the mathematical framework for the physical system responses of a $2 \mathrm{D}$ rigid cylinder arising from plane wave and monopole source excitation are given in what follows. Figure 1 shows a rigid cylinder of radius $a$ and the coordinate system to determine the acoustic pressure due to scattering by the cylinder under either incident plane wave or monopole source excitation.

\section{Plane wave excitation}

The acoustic pressure field for a rigid cylinder under incident plane wave excitation is given by ${ }^{46}$

$$
p(r, \theta)=\sum_{n=-\infty}^{\infty}\left(a_{n} \frac{J_{n}\left(k_{\mathrm{a}} r\right)}{J_{n}^{\prime}\left(k_{\mathrm{a}} a\right)}+b_{n} \frac{H_{n}\left(k_{\mathrm{a}} r\right)}{H_{n}^{\prime}\left(k_{\mathrm{a}} a\right)}\right) e^{\mathrm{i} n \theta},
$$

where $p$ is the acoustic pressure at a point of interest $(r, \theta)$ in polar coordinates. The first term in brackets on the righthand side of Eq. (1) corresponds to the incident pressure, $p_{\text {inc }}$, and the second term in brackets corresponds to the scattered pressure, $p_{\mathrm{sc}}$. The coefficients $a_{n}$ and $b_{n}$, respectively, represent the incident and scattered field coefficients, ()$^{\prime}$ is the derivative with respect to the argument, $k_{\mathrm{a}}$ is the acoustic wavenumber, $\mathrm{i}=\sqrt{-1}$ and $J_{n}, H_{n}$ are Bessel and Hankel functions of the first kind of order $n$, respectively. The boundary condition for a rigid cylinder is given by

$$
\frac{\partial}{\partial r} p(a, \theta)=0 .
$$

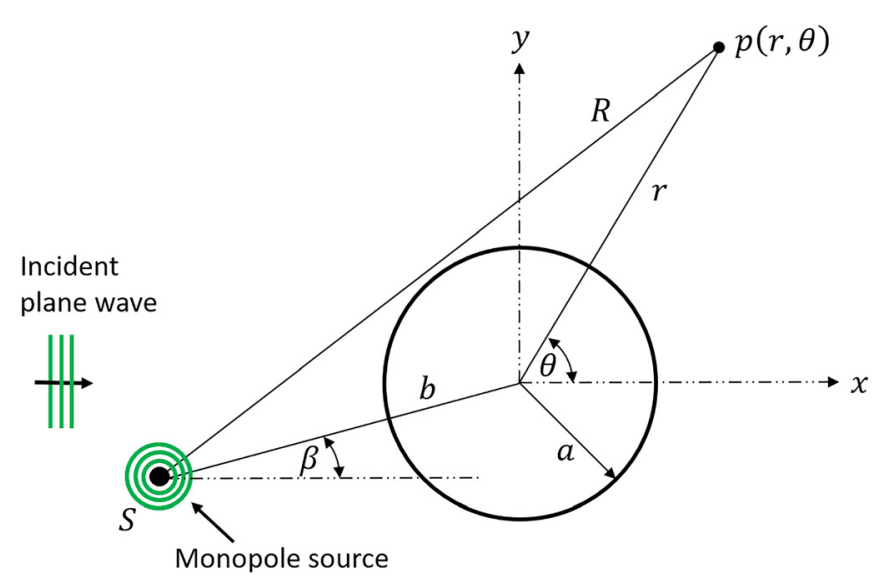

FIG. 1. (Color online) A rigid cylinder and the corresponding coordinate system for the acoustic pressure due to excitation from an incident plane wave or monopole source located at $S$. 
After substituting Eq. (1) into Eq. (2), it is possible to solve for $b_{n}$ provided $a_{n}$ is known. For plane wave excitation, these coefficients are obtained as ${ }^{46}$

$$
\begin{aligned}
& a_{n}=\mathrm{i}^{n} J_{n}^{\prime}\left(k_{\mathrm{a}} a\right), \\
& b_{n}=-\mathrm{i}^{n} J_{n}^{\prime}\left(k_{\mathrm{a}} a\right) .
\end{aligned}
$$

\section{Monopole source excitation}

A monopole source located at $S$ near the rigid cylinder as shown in Fig. 1 will emit an incident field given by

$$
p_{\text {inc }}(r, \theta)=H_{0}\left(k_{\mathrm{a}} R\right) \text {. }
$$

To determine the scattered field, we must first express the incident field in terms of the cylinder's local coordinates. This is achieved using the following addition theorem: ${ }^{46}$

$$
H_{0}\left(k_{\mathrm{a}} R\right)=\sum_{n=-\infty}^{\infty}(-1)^{n} H_{n}\left(k_{\mathrm{a}} b\right) J_{n}\left(k_{\mathrm{a}} r\right) e^{-\mathrm{i} n \beta},
$$

where $\beta$ is the angle between the monopole source and the rigid cylinder, as shown in Fig. 1. Using Eq. (6), $a_{n}$ in Eq. (1) at the boundary can be expressed as

$$
a_{n}=(-1)^{n} H_{n}\left(k_{\mathrm{a}} b\right) J_{n}^{\prime}\left(k_{\mathrm{a}} a\right) e^{-\mathrm{i} n \beta} .
$$

Using Eq. (2) to solve for $b_{n}$ yields $b_{n}=-a_{n}$. The total acoustic pressure field for a rigid cylinder under monopole source excitation becomes

$$
p(r, \theta)=H_{0}\left(k_{\mathrm{a}} R\right)+\sum_{n=-\infty}^{\infty} b_{n} \frac{H_{n}\left(k_{\mathrm{a}} r\right)}{H_{n}^{\prime}\left(k_{\mathrm{a}} a\right)} e^{\mathrm{i} n \theta} .
$$

\section{B. Potential flow model}

We herein consider a fluid of Mach number $M$ flowing past a fixed closed surface $\partial \Omega$. The Mach number is given by $M=\left|\mathbf{v}_{\infty}\right| / c_{f}$, where $\mathbf{v}_{\infty}$ is the freestream velocity and $c_{f}$ is the speed of sound in the fluid. The analysis is limited to low Mach numbers $\left(M^{2} \ll 1\right)$ where the fluid flow is modeled as potential flow. Hence the flow can be considered incompressible with $c_{f}$ assumed constant for order $M .^{47}$ Further, the velocity field is irrotational such that $\mathbf{v}(\mathbf{x})$ $=\nabla \phi_{\mathrm{v}}(\mathbf{x})$ where $\mathbf{v}(\mathbf{x})$ is the local fluid velocity, $\phi_{\mathrm{v}}(\mathbf{x})$ is the velocity potential, $\mathbf{x}$ is a point in the acoustic field $\Omega$ and $\nabla=(\partial / \partial r)+(1 / r)(\partial / \partial \theta)$ is the differential operator in cylindrical coordinates. The velocity potential for a rigid cylinder is given by ${ }^{48}$

$$
\phi_{\mathrm{v}}(\mathbf{x})=M c_{f}\left(r+\frac{a^{2}}{r}\right) \cos \theta .
$$

The potential flow is governed by the Laplace equation as follows: ${ }^{49}$

$$
\nabla^{2} \phi_{\mathrm{v}}(\mathbf{x})=0
$$

The requirement on the boundary $\partial \Omega$ is that the normal fluid velocity is zero. This can be expressed as

$$
\mathbf{n}(\mathbf{x}) \cdot \nabla \phi_{\mathrm{v}}(\mathbf{x})=0
$$

where $\mathbf{n}(\mathbf{x})$ is the unit vector normal to the boundary.

The acoustic potential $\phi_{\mathrm{a}}(\mathbf{x}, t)$ due to an acoustic excitation in the flow field is determined as follows. Provided the flow field is subject to small perturbations, the continuity and momentum equations can be linearized about the equilibrium state of the solution to Eq. (10). Utilizing these equations, it is possible to express the acoustic potential in a single equation where, for a low Mach number flow, this equation is the convected wave equation given by ${ }^{50}$

$$
\left(\frac{\partial}{\partial t}+\nabla \phi_{\mathrm{v}}(\mathbf{x}) \cdot \nabla\right)^{2} \phi_{\mathrm{a}}(\mathbf{x}, t)-c_{f}^{2} \nabla^{2} \phi_{\mathrm{a}}(\mathbf{x}, t)=0 .
$$

Note that Eq. (12) ignores terms of order $M^{2}$. The boundary condition for a rigid body is that the normal particle velocity on $\partial \Omega$ is zero. This can be satisfied by

$$
\mathbf{n}(\mathbf{x}) \cdot \nabla \phi_{\mathrm{a}}(\mathbf{x}, t)=0 .
$$

Taylor $^{51}$ derived a time transformation method that allows the convected wave equation to be reduced to the acoustic wave equation. Using the following transformations:

$$
\mathbf{X}=\mathbf{x}, \quad T=t+\phi_{\mathrm{v}}(\mathbf{x}) / c_{f}^{2},
$$

and assuming a time harmonic solution such that the acoustic potential in the transformed domain is given by $\hat{\phi}_{\mathrm{a}}(\mathbf{X}, T)$ $=\hat{\phi}_{\mathrm{h}}(\mathbf{X} ; \omega) e^{-\mathrm{i} \omega T}$, Eq. (12) reduces to the Helmholtz equation in transformed coordinates as follows:

$$
\left(\nabla^{2}+k_{\mathrm{a}}^{2}\right) \hat{\phi}_{\mathrm{h}}(\mathbf{X} ; \omega)=0
$$

where $\omega$ is the excitation frequency and $\hat{\phi}_{\mathrm{h}}(\mathbf{X} ; \omega)$ is the time harmonic acoustic potential in the transformed domain.

The solution to Eq. (15) for an incident plane wave or monopole source excitation can be easily solved using the scattering theory described in Sec. II. A solution for either excitation is given by

$$
\hat{\phi}_{\mathrm{a}}(\mathbf{X}, T)=\Phi(\mathbf{X}) e^{-\mathrm{i} \omega T},
$$

where $\Phi(\mathbf{X})$ is the solution that satisfies Eq. (15) in the transformed domain. Equation (16) is then transformed back to the original variables which yields

$$
\phi_{\mathrm{a}}(\mathbf{x}, t)=\Phi(\mathbf{x}) \exp \left(-\mathrm{i} \omega \phi_{\mathrm{v}}(\mathbf{x}) / c_{f}^{2}\right) e^{-\mathrm{i} \omega t} .
$$

The acoustic potential in Eq. (17) is related to the acoustic pressure via the Bernoulli equation as follows: ${ }^{49}$

$$
p(\mathbf{x}, t)=-\bar{\rho}_{f}\left(\frac{\partial \phi_{\mathrm{a}}(\mathbf{x}, t)}{\partial t}+\nabla \phi_{\mathrm{v}}(\mathbf{x}) \cdot \nabla \phi_{\mathrm{a}}(\mathbf{x}, t)\right),
$$

where $\bar{\rho}_{f}$ is the freestream mean density. The resultant acoustic pressure, correct to order $M^{2}$, can now be written as $^{52}$ 


$$
\begin{aligned}
p(\mathbf{x}, t)= & -\bar{\rho}_{f}\left(-\mathrm{i} \omega \Phi(\mathbf{x})+\nabla \phi_{\mathrm{v}}(\mathbf{x}) \cdot \nabla \Phi(\mathbf{x})\right) \\
& \times \exp \left(-\mathrm{i} \omega \phi_{\mathrm{v}}(\mathbf{x}) / c_{f}^{2}\right) e^{-\mathrm{i} \omega t} .
\end{aligned}
$$

In the absence of potential flow, i.e., $M=0$, Eq. (19) reduces to the acoustic pressure in a stationary fluid given by $p(\mathbf{x}, t)$ $=-\bar{\rho}_{f}(-\mathrm{i} \omega \Phi(\mathbf{x})) e^{-\mathrm{i} \omega t}$. It is important to note that Eq. (19) does not provide information about the phase due to the effect of the fluid flow between the location of a monopole source and the field point. ${ }^{51}$ This phase information is essential when considering multiple monopole sources and their resultant complex pressure at a given field point. To account for the phase between a source location and a field point, a modification must be made to Eq. (19) as follows: ${ }^{47,51}$

$$
\begin{aligned}
p(\mathbf{x}, t)= & -\bar{\rho}_{f}\left(-\mathrm{i} \omega \Phi(\mathbf{x})+\nabla \phi_{\mathrm{v}}(\mathbf{x}) \cdot \nabla \Phi(\mathbf{x})\right) \\
& \times \exp \left(\mathrm{i} \omega\left(\phi_{\mathrm{v}}\left(\mathbf{x}_{s}\right)-\phi_{\mathrm{v}}(\mathbf{x})\right) / c_{f}^{2}\right) e^{-\mathrm{i} \omega t},
\end{aligned}
$$

where $\mathbf{x}_{\mathrm{s}}$ corresponds to the location of the source. Note that plane wave excitation has an undefined source location which causes the additional term in Eq. (20) corresponding to $\phi_{\mathrm{v}}\left(\mathbf{x}_{S}\right)$ to become zero, resulting in Eq. (19) as before.

\section{ACTIVE CLOAKING METHODOLOGY}

Figure 2 outlines the active control configuration used in our study. An incident acoustic field corresponding to either a plane wave or a single primary monopole source located $4 a$ upstream impinges on a rigid cylinder of radius $a$. Monopole control sources to generate the secondary field are circumferentially arranged around the cylinder at a radial distance of $2 a$, with microphone error sensors, also circumferentially arranged, located at $3 a$.

ANC typically reduces the total acoustic pressure at the error sensor locations. ${ }^{41}$ Active noise cloaking requires a

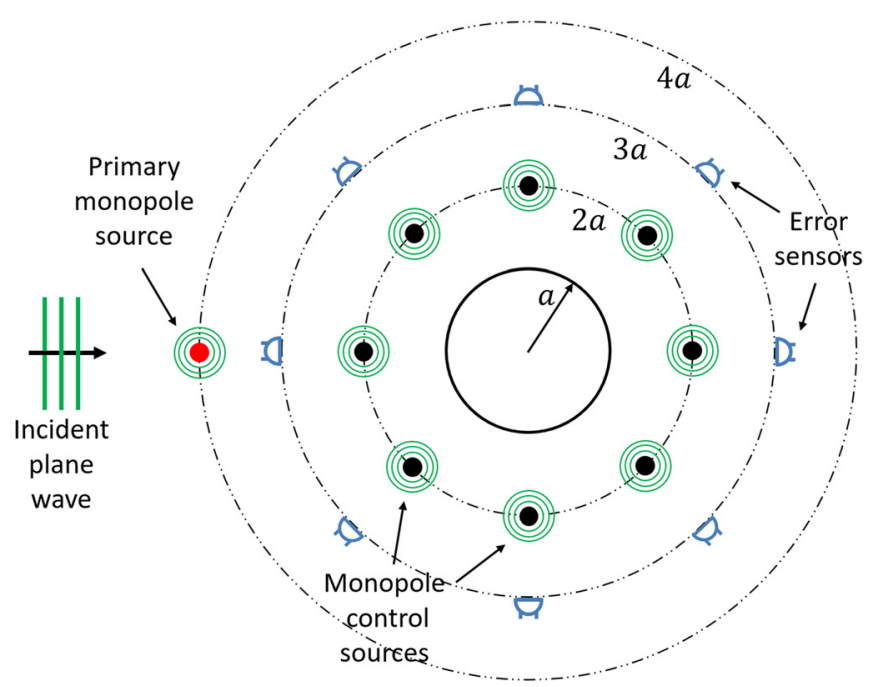

FIG. 2. (Color online) Control arrangement for active cloaking of a rigid cylinder of radius $a$. The incident field corresponds to a plane wave or a single primary monopole source located at $4 a$ upstream of the cylinder. Monopole control sources and error sensors are located circumferentially around the cylinder at $2 a$ and $3 a$, respectively. modification to the cost function to consider all acoustic pressure fields excluding the incident field at each of the error sensors. The quadratic cost function to be minimized corresponds to the sum of the squared acoustic pressures at the error sensors and is given by

$$
J=\mathbf{e}_{\mathrm{cl}}^{\mathrm{H}} \mathbf{e}_{\mathrm{cl}},
$$

where the superscript $\mathrm{H}$ denotes the Hermitian transpose operator. The error vector is defined as

$$
\mathbf{e}_{\mathrm{cl}}=\mathbf{p}_{\mathrm{sc}}+\mathbf{Z q}
$$

where $\mathbf{p}_{\mathrm{sc}}$ is a vector denoting the primary scattered acoustic pressure at the error sensors due to an incident field impinging on the body, $\mathbf{Z}$ is an $L \times W$ matrix of complex transfer functions representing the secondary field at $L$ error sensor locations arising from $W$ monopole control sources interacting with the body, and $\mathbf{q}$ is the vector of $W$ monopole control source strengths to be optimized such that the complex pressure given by Eq. (22) is minimized. The elements of the matrix $Z$ are given by the following equation:

$$
Z_{i j}=H_{0}\left(k_{\mathrm{a}} R_{i j}\right)+\sum_{n=-\infty}^{\infty} b_{n} \frac{H_{n}\left(k_{\mathrm{a}} r_{i}\right)}{H_{n}^{\prime}\left(k_{\mathrm{a}} a\right)} e^{\mathrm{i} n \theta_{i}},
$$

where the subscripts $i$ and $j$, respectively, refer to the $i$ th error sensor and the $j$ th monopole control source. $R_{i j}, r_{i}$, and $\theta_{i}$ are the distances and angle shown in Fig. 1 for the $i$ th error sensor relative to the $j$ th monopole control source and the rigid body. Based on a least-mean-square adaptive feedforward algorithm, ${ }^{53}$ the optimal control source strengths are determined by differentiating Eq. (21) with respect to the real and imaginary components of the complex control sources and equating the resulting expressions to zero, which yields

$$
\mathbf{q}=-\left[\mathbf{Z}^{\mathrm{H}} \mathbf{Z}\right]^{-1} \mathbf{Z}^{\mathrm{H}} \mathbf{p}_{\mathrm{sc}}
$$

It should be noted that a limitation of the current active acoustic cloaking approach is the requirement of a priori knowledge of the incident field. Strategies to overcome this hurdle have been presented by various authors. ${ }^{32-35,54}$

\section{RESULTS AND DISCUSSION}

Results are presented for a rigid cylinder of radius $a=0.6 \mathrm{~m}$ in air of density $\rho_{f}=1.2 \mathrm{~kg} / \mathrm{m}^{3}$ and speed of sound $c_{f}=340 \mathrm{~m} / \mathrm{s}$. Note that the analysis is limited to low Mach number flow $(M<0.3)$. The number of control sources and error sensors were selected such that a percentage error function given by

$$
\Delta=\frac{|| \mathbf{p}_{\text {inc }}|-| \mathbf{p}_{\mathrm{cl}}||}{\left|\mathbf{p}_{\text {inc }}\right|} \times 100 \%
$$

gives a predefined percentage error of $\Delta<1 \%$. In Eq. (25), $\mathbf{p}_{\text {inc }}$ is the incident pressure given by the first term on the right-hand side of Eq. (1) for incident plane wave excitation or corresponds to Eq. (5) for primary monopole source 
excitation. $\mathbf{p}_{\mathrm{cl}}$ is the cloaked pressure given by the resultant acoustic pressure corresponding to $\mathbf{p}_{\text {inc }}+\mathbf{p}_{\mathrm{sc}}+\mathbf{Z q}$ after implementation of the active cloaking procedure. The process initially considered a large number of error sensors such that increasing the number of sensors had no effect on the resultant error. The number of control sources was gradually reduced ensuring that the error remained less than $1 \%$. After the minimum number of control sources was identified, the
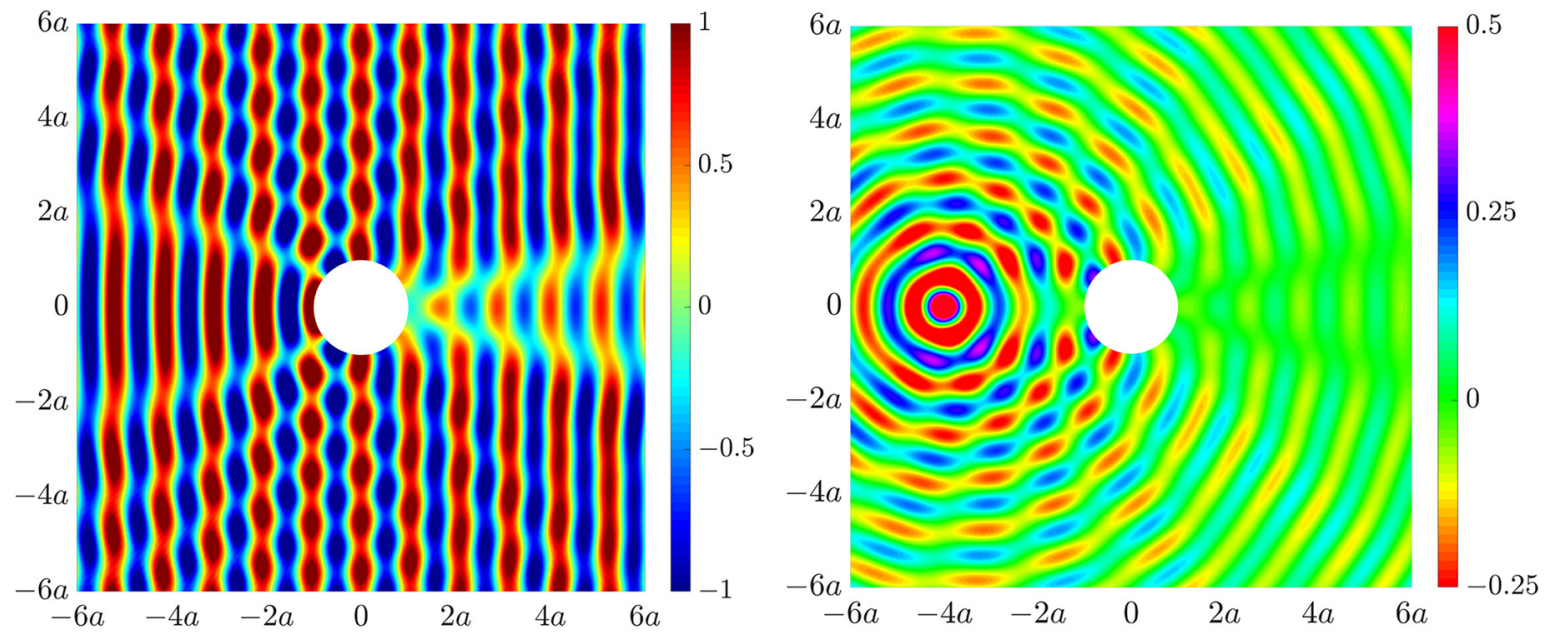

(a)
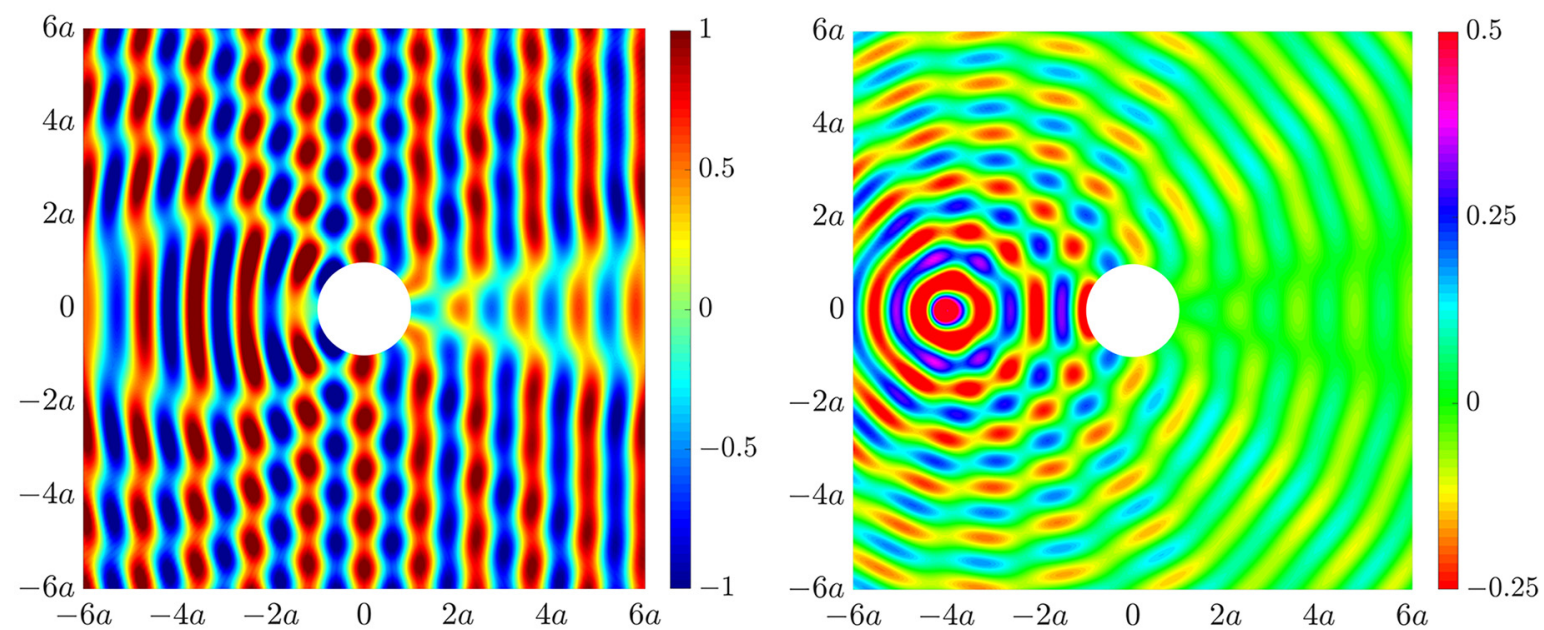

(b)
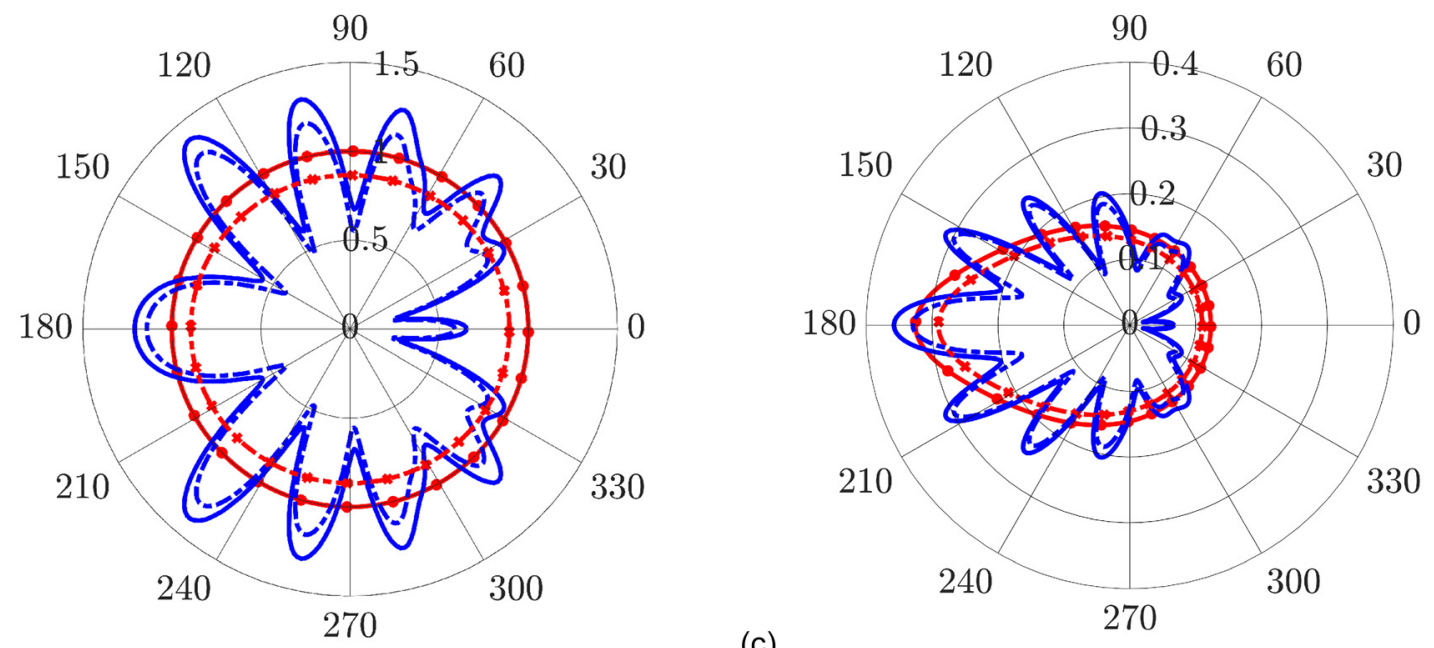

(c)

FIG. 3. (Color online) Primary acoustic pressure field (in Pascal) arising from scattering by a rigid cylinder due to an incident plane wave (left column) and a primary monopole source (right column) at a non-dimensionalized frequency $k_{\mathrm{a}} a=6$, (a) in the absence of convected flow $(M=0)$, (b) in the presence of convected flow $(M=0.12)$, (c) directivities of the incident field in the absence of convected flow (circle-marked solid red line) and in the presence of convected flow (cross-marked dashed-dotted red line), directivities of the primary acoustic field in the absence of convected flow (solid blue line) and in the presence of convected flow (dashed-dotted blue line), at a radial distance of $r=3 a$. 
number of error sensors was reduced, still maintaining the resultant error less than $1 \%$.

Results are herein presented at a non-dimensionalized excitation frequency of $k_{\mathrm{a}} a=6$. Figure 3(a) shows the primary acoustic pressure field due to an incident plane wave (left) and primary monopole source (right) impinging on the cylinder in the absence of potential flow $(M=0)$. Scattering in both the forward and backscatter regions can
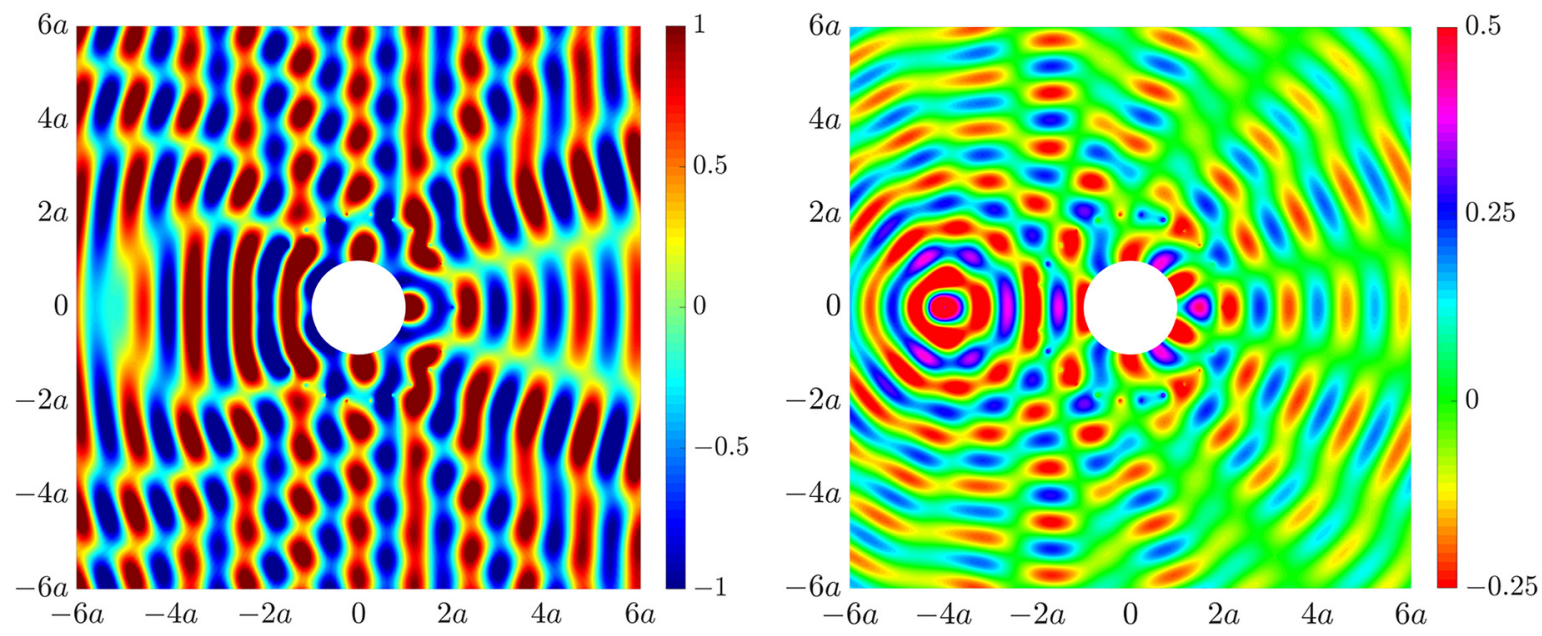

(a)
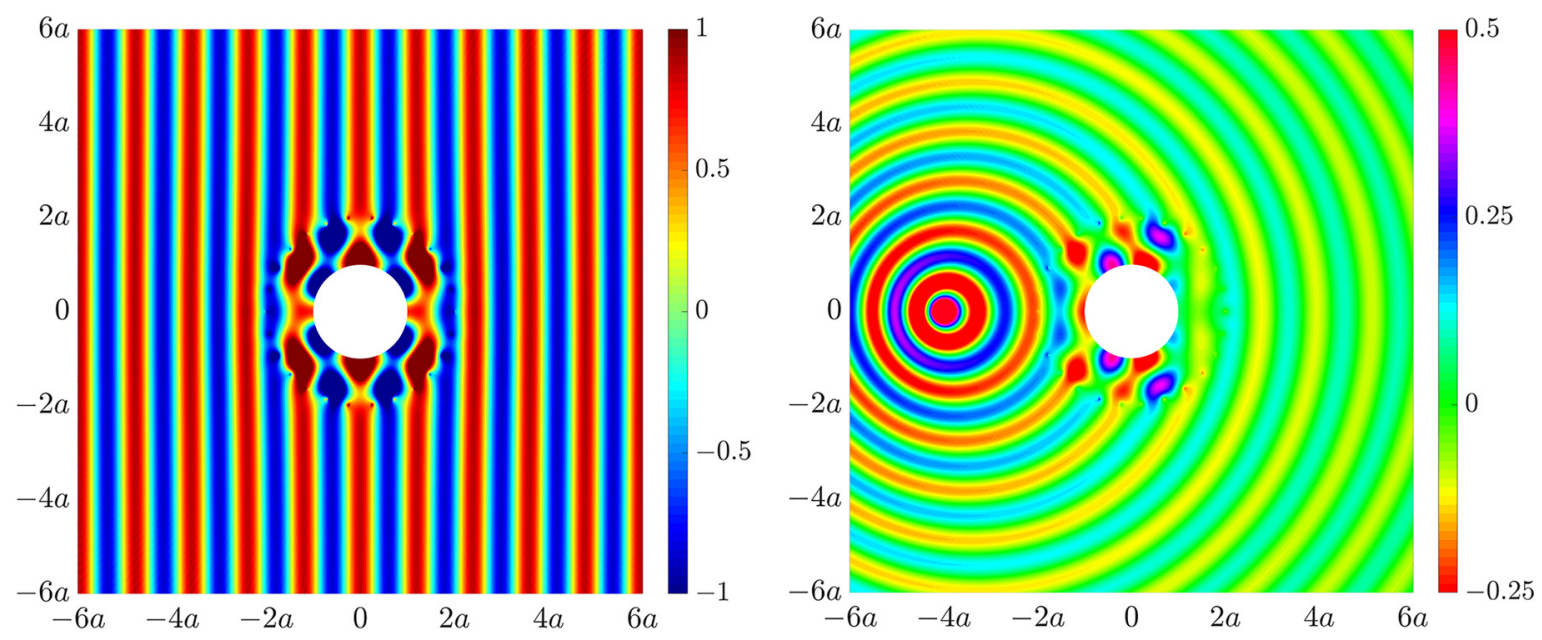

(b)
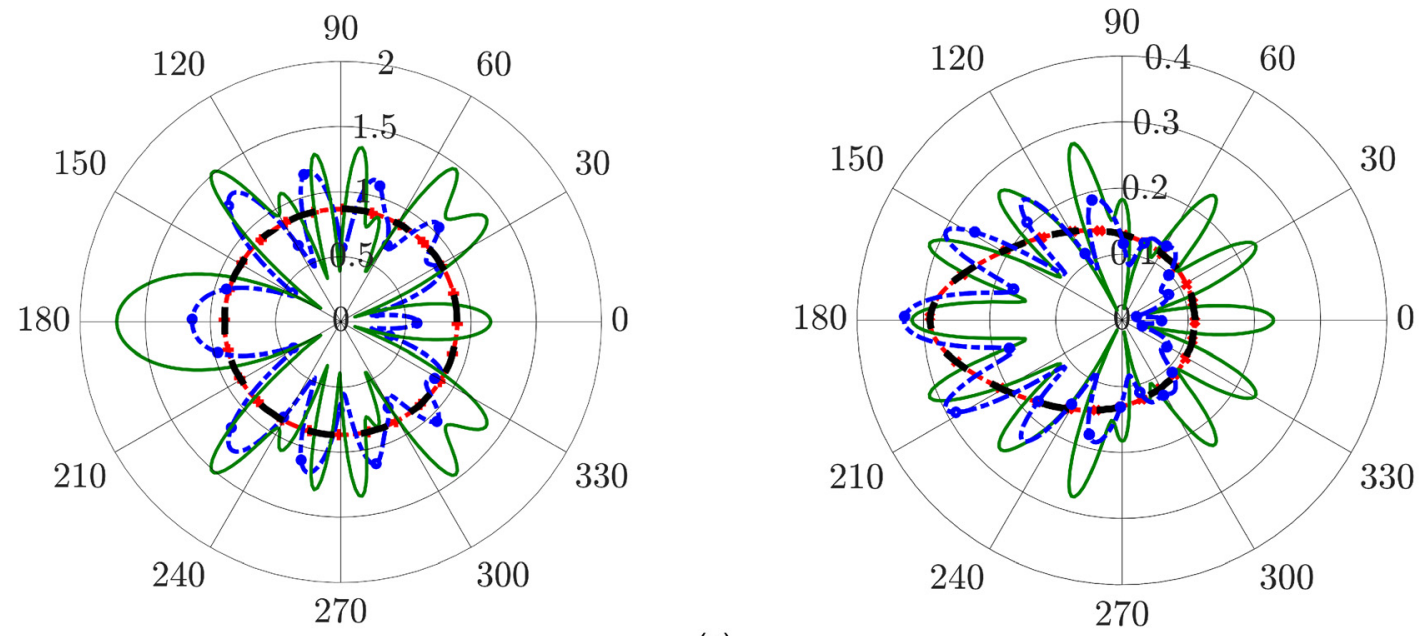

(c)

FIG. 4. (Color online) Acoustic pressure (in Pascal) at a non-dimensionalized frequency $k_{\mathrm{a}} a=6$ in a convected flow field $(M=0.12)$ due to excitation from an incident plane wave (left column) and a primary monopole source (right column), (a) controlled acoustic field neglecting convected flow, (b) controlled acoustic field accounting for convected flow, (c) directivities of the incident field (cross-marked dashed-dotted red line), primary acoustic field (circle-marked dashed-dotted blue line), controlled acoustic field neglecting convected flow (solid green line), and controlled acoustic field accounting for convected flow (dashed black line), at a radial distance of $r=3 a$. 
be observed. Figure 3(b) presents results similar to Fig. 3(a) for a convected flow field of $M=0.12$. The additional effect of the convected flow field on the acoustic responses is not significant compared to the absence of flow, with the greatest impact occurring in the backscatter region for both excitation cases. The Doppler effect can be seen with contraction and expansion of the wavelength of the acoustic field due to motion of the fluid medium. Figure 3(c) presents the
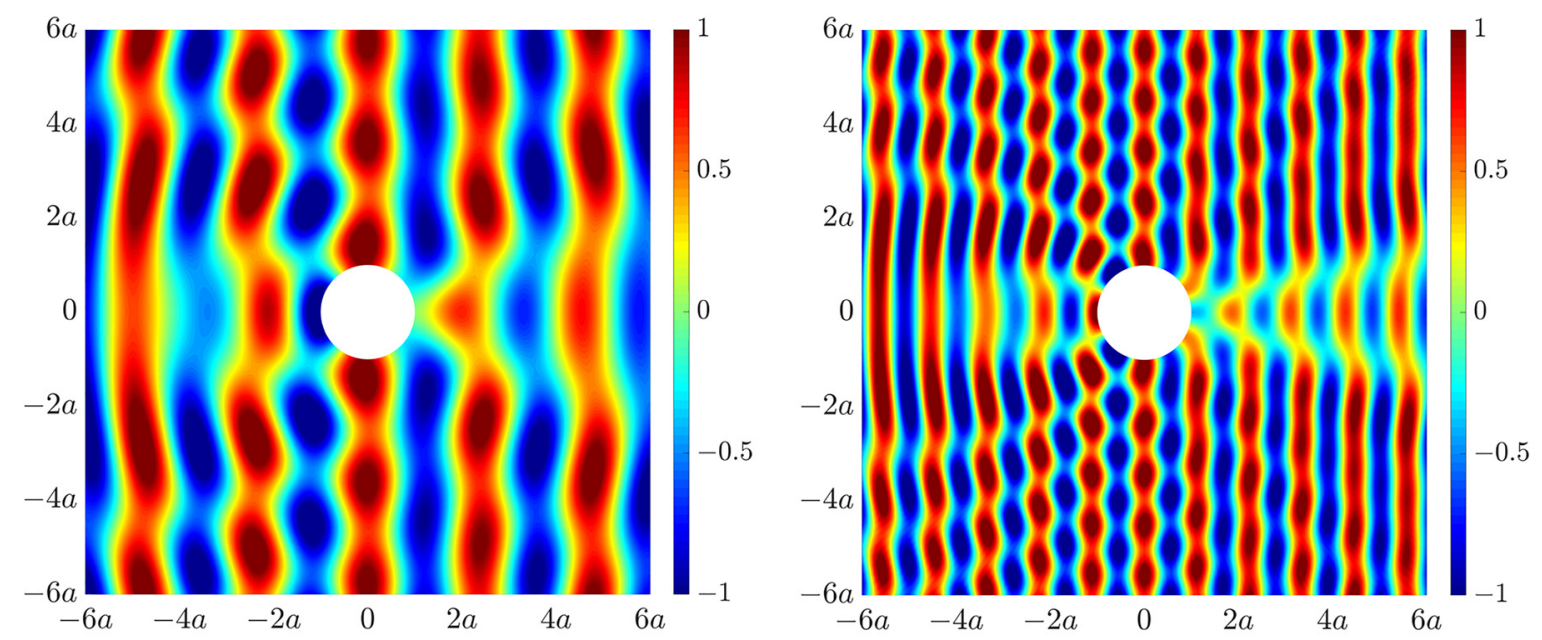

(a)
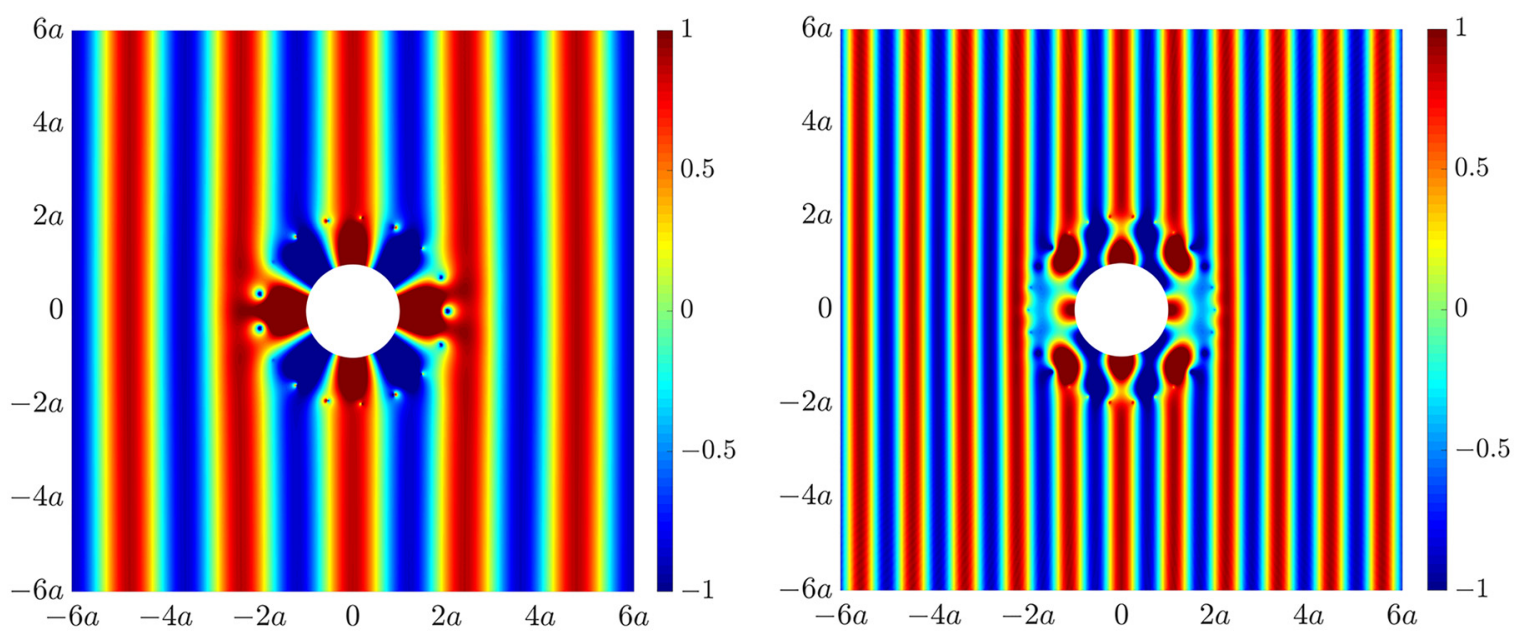

(b)
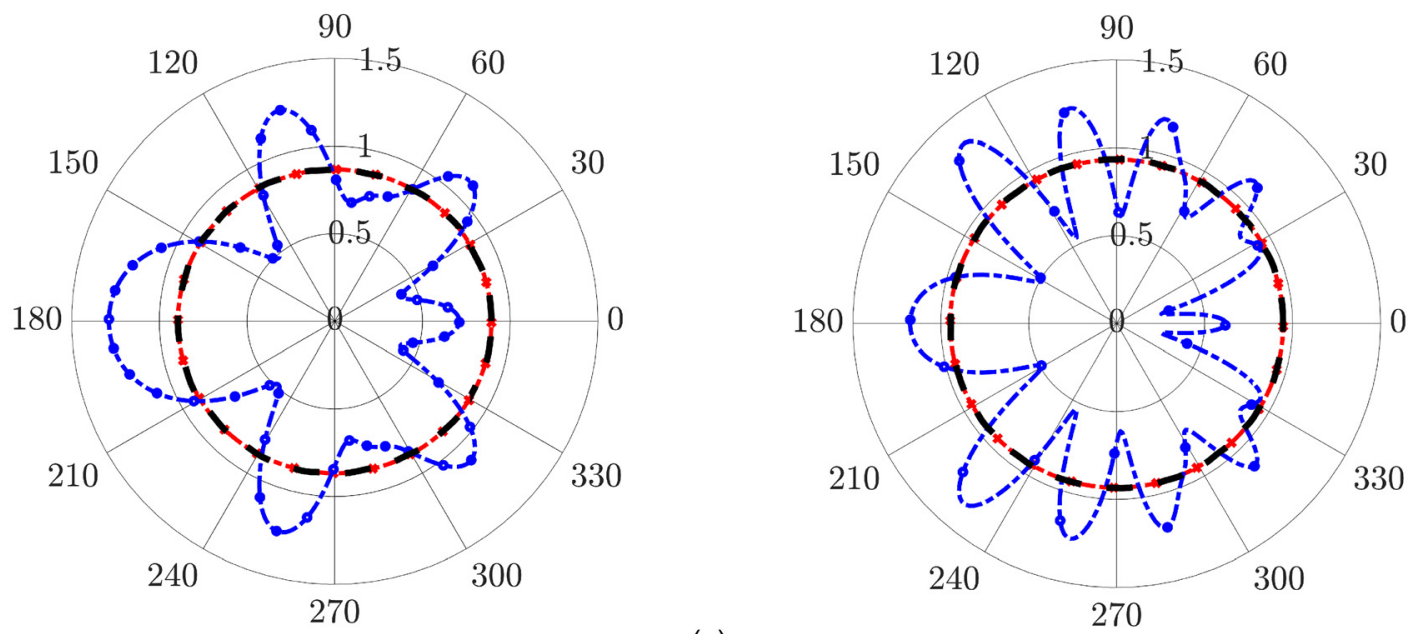

(c)

FIG. 5. (Color online) Acoustic pressure (in Pascal) arising from scattering by a rigid cylinder due to an incident plane wave at a non-dimensionalized frequency $k_{\mathrm{a}} a=3$ in a convected flow field of $M=0.12$ (left column) and at a non-dimensionalized frequency $k_{\mathrm{a}} a=6$ in a convected flow field of $M=0.06$ (right column), (a) primary acoustic field, (b) controlled acoustic field, (c) directivities of the incident field (cross-marked dashed-dotted red line), primary acoustic field (circle-marked dashed-dotted blue line), and controlled acoustic field (dashed black line), at a radial distance of $r=3 a$. 
directivities of the incident field and the primary acoustic field due to scattering by a plane wave (left) and primary monopole source (right), at a radial distance of $r=3 a$. Directivity is the absolute acoustic pressure evaluated at a specific radial distance for all field points along the circumference. In the absence of potential flow, the directivity of the incident field is unity for the plane wave [Fig. 3(c), left], and displays the greatest magnitude in the backscatter region in line with the source location of the primary monopole [Fig. 3(c), right]. Figure 3(c) shows that the presence of potential flow does not significantly modify the shape of the directivities associated with the incident and scattered fields for either excitation case, with the main effect corresponding to a reduction in magnitude.

Figure 4 presents results for active acoustic cloaking in a convected flow field of $M=0.12$, whereby the effects of convection have been neglected or included in the cloaking process. Using the procedure to select the minimum number of control sources and error sensors such that $\Delta<1 \%$, the control configuration in Fig. 2 utilized 26 control sources and 40 error sensors. The same number of control sources and error sensors for excitation from an incident plane wave or a primary monopole source is attributed to the same excitation frequency used for both primary excitation cases, whereby the number of control sources and error sensors is only dependent on the acoustic wavelength as discussed previously. ${ }^{31}$ Figure 4(a) shows the controlled field arising from application of active cloaking using the optimal control source strengths derived in the absence of flow, clearly highlighting that cloaking is not achieved when convection effects are neglected. Further, constructive interference between the primary and secondary acoustic fields increases the total acoustic response in regions around and particularly downstream of the cylinder. Figure 4(b) presents the controlled field with active cloaking now accounting for the convected flow field. Beyond the radial location of the control sources, the resultant acoustic field resembles that of the incident field associated with a plane wave (left) or single monopole source (right). Figure 4(c) presents the directivities of the incident plane wave (left) and primary monopole source (right) in a convected flow field. Also included are the directivities of the primary acoustic field, the controlled field when convection is neglected, and the controlled field when convected flow is taken into account. All directivities were obtained at a radial distance of $r=3 a$. For both excitation cases, when convected flow is included in the cloaking process, the directivity of the cloaked field is identical to the directivity of the incident field. However, when convection is neglected, the resultant directivity is shown to deviate significantly as well as increase in directional regions, highlighting the need to account for the effects of convection for acoustic cloaking in the presence of flow.

Figure 5 presents results at a different Mach number and frequency. The left column considers a convected flow field of $M=0.12$ at a non-dimensionalized frequency of $k_{\mathrm{a}} a=3$, while the right column considers a flow field of $M=0.06$ at a non-dimensionalized frequency of $k_{\mathrm{a}} a=6$. Figure 5(a) presents the uncontrolled responses for these cases under incident plane wave excitation. The reduction in frequency (left column) shows an increase in the corresponding acoustic wavelength and less pronounced scattering, whereas the decrease in the Mach number (right column) exhibits less expansion and contraction of the acoustic wavelength attributed to the reduced Doppler effect induced by the flow, particularly in the backscatter region. Figure 5(b) shows the cloaked acoustic field when accounting for the effects of convected flow. When the non-dimensionalized frequency is reduced to $k_{\mathrm{a}} a=3$ (left column), the minimum number of control sources and error sensors such that $\Delta<1 \%$ is reduced to 17 control sources and 30 error sensors. When only the Mach number is reduced to $M=0.06$ (right column), the number of control sources and error sensors is unchanged. This indicates that the cloaking parameters are largely insensitive to variation in Mach number for potential flow. Figure 5(c) presents the directivities of the incident plane wave, primary acoustic field, and the controlled acoustic field at a radial distance of $r=3 a$. The controlled field directivity is identical to the incident directivity after active acoustic cloaking in a convected flow field has been implemented.

\section{SUMMARY}

Active acoustic cloaking has been demonstrated for a rigid cylinder under excitation from an incident plane wave or monopole source in a convected flow field. The control configuration was comprised of control sources and error sensors located circumferentially around the cylinder. Convected flow was observed to have little effect on the primary acoustic responses for either excitation case. However, it was shown that ignoring the effects of convected flow can result in amplification of the controlled acoustic field, attributed to constructive interference between the primary and secondary fields, respectively, associated with the incident excitation and control sources. When convection effects were taken into account, the cloaked acoustic field resembled the incident field beyond the location of the control sources.

\section{ACKNOWLEDGMENTS}

The authors would like to thank Dr. Hyuck Chung for his helpful discussion on applying scattering theory in conjunction with Taylor's transformations.

${ }^{1}$ J. B. Pendry, D. Schurig, and D. R. Smith, "Controlling electromagnetic fields," Science 312, 1780-1782 (2006).

${ }^{2}$ W. Cai, U. K. Chettiar, A. V. Kildishev, and V. M. Shalaev, "Optical cloaking with metamaterials," Nat. Photon. 1, 224-227 (2007).

${ }^{3}$ B. Zhang, H. Chen, B. I. Wu, Y. Luo, L. Ran, and J. A. Kong, "Response of a cylindrical invisibility cloak to electromagnetic waves," Phys. Rev. B 76, 121101 (2007).

${ }^{4}$ A. Alù and N. Engheta, "Plasmonic and metamaterial cloaking: Physical mechanisms and potentials," J. Opt. A Pure Appl. Opt. 10, 093002 (2008).

${ }^{5}$ M. Farhat, S. Guenneau, A. B. Movchan, and S. Enoch, "Achieving invisibility over a finite range of frequencies," Opt. Express 16, 5656-5661 (2008).

${ }^{6}$ A. Alù, "Mantle cloak: Invisibility induced by a surface," Phys. Rev. B 80, 245115 (2009).

${ }^{7}$ Y. Cheng, F. Yang, J. Y. Xu, and X. J. Liu, "A multilayer structured acoustic cloak with homogeneous isotropic materials," Appl. Phys. Lett. 92, 151913 (2008). 
${ }^{8}$ A. N. Norris, "Acoustic cloaking theory," Proc. Royal Soc. A 464, 2411-2434 (2008).

${ }^{9}$ S. A. Cummer and D. Schurig, "One path to acoustic cloaking," New J. Phys. 9, 1-8 (2007).

${ }^{10}$ D. Torrent and J. Sánchez-Dehesa, “Acoustic cloaking in two dimensions: A feasible approach,” New J. Phys. 10, 063015 (2008).

${ }^{11} \mathrm{H}$. Chen and C. T. Chan, "Acoustic cloaking in three dimensions using acoustic metamaterials," Appl. Phys. Lett. 91, 183518 (2007).

${ }^{12}$ S. A. Cummer, B.-I. Popa, D. Schurig, D. R. Smith, J. B. Pendry, M. Rahm, and A. Starr, "Scattering theory derivation of a 3D acoustic cloaking shell," Phys. Rev. Lett. 100, 024301 (2008).

${ }^{13} \mathrm{D}$. Torrent and J. Sánchez-Dehesa, “Acoustic metamaterials for new twodimensional sonic devices," New J. Phys. 9, 1-13 (2007).

${ }^{14}$ B.-I. Popa and S. A. Cummer, "Cloaking with optimized homogeneous anisotropic layers," Phys. Rev. A 79, 023806 (2009).

${ }^{15}$ J. B. Pendry and J. Li, "An acoustic metafluid: Realizing a broadband acoustic cloak," New J. Phys. 10, 115032 (2008).

${ }^{16} \mathrm{Y}$. Cheng and X. Liu, "Resonance effects in broadband acoustic cloak with multilayered homogeneous isotropic materials," Appl. Phys. Lett. 93, 071903 (2008).

${ }^{17}$ S. Zhang, C. Xia, and N. Fang, "Broadband acoustic cloak for ultrasound waves," Phys. Rev. Lett. 106, 024301 (2011).

${ }^{18}$ B.-I. Popa, L. Zigoneanu, and S. A. Cummer, "Experimental acoustic ground cloak in air" Phys. Rev. Lett. 106, 253901 (2011).

${ }^{19}$ L. Zigoneanu, B.-I. Popa, and S. A. Cummer, "Three-dimensional broadband omnidirectional acoustic ground cloak," Nat. Mater. 13, 352-355 (2014).

${ }^{20}$ Y. Cheng and X. J. Liu, "Three dimensional multilayered acoustic cloak with homogeneous isotropic materials," Appl. Phys. A 94, 25-30 (2009).

${ }^{21}$ M. Farhat, S. Enoch, S. Guenneau, and A. B. Movchan, "Broadband cylindrical acoustic cloak for linear surface waves in a fluid," Phys. Rev. Lett. 101, 134501 (2008).

${ }^{22}$ Y. Chen, X. Liu, and G. Hu, "Latticed pentamode acoustic cloak," Sci. Rep. 5, 15745 (2015).

${ }^{23}$ Y. Chen, M. Zheng, X. Liu, Y. Bi, Z. Sun, P. Xiang, J. Yang, and G. Hu, "Broadband solid cloak for underwater acoustics," Phys. Rev. B 95, 180104 (2017).

${ }^{24}$ F. Guevara Vasquez, G. W. Milton, and D. Onofrei, "Active exterior cloaking for the 2D Laplace and Helmholtz equations," Phys. Rev. Lett. 103, 073901 (2009).

${ }^{25}$ F. Guevara Vasquez, G. W. Milton, D. Onofrei, and P. Seppecher, "Transformation elastodynamics and active exterior acoustic cloaking," in Acoustic Metamaterials (Springer, New York, 2013), pp. 289-318.

${ }^{26}$ F. Guevara Vasquez, G. W. Milton, and D. Onofrei, "Exterior cloaking with active sources in two dimensional acoustics," Wave Motion 48, 515-524 (2011).

${ }^{27}$ Y. I. Bobrovnitskii, "A new solution to the problem of an acoustically transparent body," Acoust. Phys. 50, 647-650 (2004).

${ }^{28}$ Y. I. Bobrovnitskii, "A new impedance-based approach to analysis and control of sound scattering," J. Sound Vib. 297, 743-760 (2006).

${ }^{29}$ M. Rajabi and A. Mojahed, "Active acoustic cloaking spherical shells," Acta Acust. Acust. 104, 5-12 (2018).

${ }^{30}$ J. Cheer, "Active control of scattered acoustic fields: Cancellation, reproduction and cloaking," J. Acoust. Soc. Am. 140, 1502-1512 (2016).

${ }^{31}$ D. Eggler, H. Chung, F. Montiel, J. Pan, and N. Kessissoglou, "Active noise cloaking of 2D cylindrical shells," Wave Motion 87, 106-122 (2019).
${ }^{32}$ E. Friot and C. Bordier, "Real-time active suppression of scattered acoustic radiation," J. Sound Vib. 278, 563-580 (2004).

${ }^{33}$ E. Friot, R. Guillermin, and M. Winninger, "Active control of scattered acoustic radiation: A real-time implementation for a three-dimensional object," Acta Acust. Acust. 92, 278-288 (2006), available at https:// www.ingentaconnect.com/content/dav/aaua/2006/00000092/00000002/ art00010? crawler=true.

${ }^{34} \mathrm{~N}$. Han, S. Feng, and X. Qiu, "Active control of one-dimension impulsive reflection based on a prediction method," J. Acoust. Soc. Am. 127, 1193-1196 (2010).

${ }^{35}$ N. Han, X. Qiu, and S. Feng, "Active control of three-dimension impulsive scattered radiation based on a prediction method," Mech. Syst. Signal Process. 30, 267-273 (2012).

${ }^{36}$ A. N. Norris, F. A. Amirkulova, and W. J. Parnell, "Active elastodynamic cloaking," Math. Mech. Solids 19, 603-625 (2014).

${ }^{37}$ G. Futhazar, W. J. Parnell, and A. N. Norris, "Active cloaking of flexural waves in thin plates," J. Sound Vib. 356, 1-19 (2015).

${ }^{38}$ J. O'Neill, Ö. Selsil, R. C. McPhedran, A. B. Movchan, and N. V. Movchan, "Active cloaking of inclusions for flexural waves in thin elastic plates," Q. J. Mech. Appl. Math. 68, 263-288 (2015).

${ }^{39}$ J. O’Neill, Ö. Selsil, R. C. McPhedran, A. B. Movchan, N. V. Movchan, and C. Henderson Moggach, "Active cloaking of resonant coated inclusions for waves in membranes and Kirchhoff plates," Q. J. Mech. Appl. Math. 69, 115-159 (2016).

${ }^{40}$ J. O’Neill, Ö. Selsil, S. G. Haslinger, N. V. Movchan, and R. V. Craster, "Active cloaking for finite clusters of pins in Kirchhoff plates," SIAM J. Appl. Math. 77, 1115-1135 (2017).

${ }^{41}$ P. A. Nelson and S. J. Elliott, Active Control of Sound (Academic Press, Cambridge, MA, 1991).

${ }^{42} \mathrm{X}$. Huang, S. Zhong, and O. Stalnov, "Analysis of scattering from an acoustic cloak in a moving fluid," J. Acoust. Soc. Am. 135, 2571-2580 (2014).

${ }^{43} \mathrm{X}$. Huang, S. Zhong, and X. Liu, "Acoustic invisibility in turbulent fluids by optimised cloaking," J. Fluid Mech. 749, 460-477 (2014).

${ }^{44} \mathrm{H}$. Ryoo and W. Jeon, "Effect of compressibility and non-uniformity in flow on the scattering pattern of acoustic cloak," Sci. Rep. 7, 2125 (2017).

${ }^{45} \mathrm{U}$. Iemma and G. Palma, "Convective correction of metafluid devices based on Taylor transformation," J. Sound Vib. 443, 238-252 (2019).

${ }^{46} \mathrm{P}$. A. Martin, Multiple Scattering: Interaction of Time-harmonic Waves with $N$ Obstacles (Cambridge University Press, London, 2006).

${ }^{47}$ G. K. Batchelor, An Introduction to Fluid Dynamics (Cambridge University Press, London, 2000).

${ }^{48} \mathrm{M}$. Van Dyke, Perturbation Methods in Fluid Mechanics (Annotated edition, The Parabolic Press, CA, 1975).

${ }^{49}$ A. Agarwal and A. P. Dowling, "Low-frequency acoustic shielding by the silent aircraft airframe," AIAA J. 45, 358-365 (2007).

${ }^{50} \mathrm{M}$. Howe, "The generation of sound by aerodynamic sources in an inhomogeneous steady flow," J. Fluid Mech. 67, 597-610 (1975).

${ }^{51} \mathrm{~K}$. Taylor, "A transformation of the acoustic equation with implications for wind-tunnel and low-speed flight tests," Proc. Royal Soc. London A 363, 271-281 (1978).

${ }^{52}$ W. R. Wolf and S. K. Lele, "Fast acoustic scattering predictions with nonuniform potential flow effects," J. Braz. Soc. Mech. Sci. Eng. 35, 407-418 (2013).

${ }^{53}$ B. Widrow and S. Stearns, Adaptive Signal Processing (Prentice-Hall, Englewood Cliffs, NJ, 1985).

${ }^{54}$ D. A. Miller, "On perfect cloaking," Opt. Express 14, 12457-12466 (2006). 